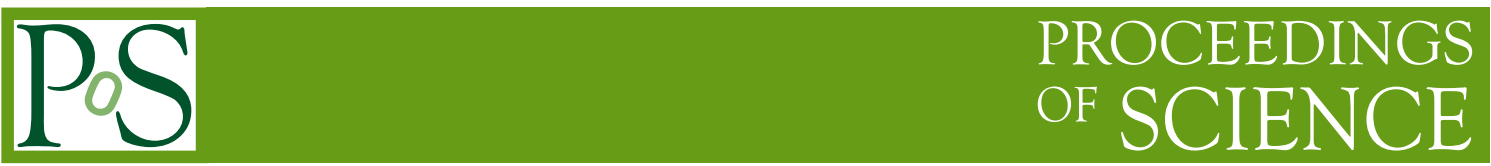

\title{
Status and perspectives of the Euclid mission
}

\author{
Alessandro Renzi ${ }^{* \dagger}$ \\ INFN-Padova, Via Marzolo 8, I-35131 Padova, Italy \\ E-mail: alessandro.renzi@pd.infn.it
}

Euclid is the M2 mission of ESA's Cosmic Vision program dedicated to the study of the dark universe: Dark Matter and Dark Energy, with launch scheduled for 2022. Euclid will observe 15,000 square degrees of extragalactic sky in the visible band with resolution of $0.1 \operatorname{arcsec}$ (VIS), in IR photometry for the Y, J, H bands and in slitless spectroscopy between 1 and 2 microns (NISP). Euclid will be able to measure the gravitationally induced distortion of the apparent shapes of about one billion of galaxies (Weak Lensing), and Galaxy Clustering (BAO and RSD), using several tens of millions of spectroscopic redshift determinations and billions of photometric redshifts. After a short introduction to the problem of the accelerated expansion of the Universe and Dark Energy the talk will illustrate the scientific objectives of Euclid and give an update of its current status, along with the expected results and foreseen precision and accuracy.

European Physical Society Conference on High Energy Physics - EPS-HEP2019 -

10-17 July, 2019

Ghent, Belgium

\footnotetext{
${ }^{*}$ Speaker.

${ }^{\dagger}$ On behalf of the Euclid Consortium.
} 


\section{Introduction}

From the Planck satellite's Cosmic Microwave Background (CMB) data analysis [1] we know that the energy content of the Universe is composed of $\sim 68 \%$ of Dark Energy (DE), $\sim 27 \%$ of Dark Matter (DM) and $\sim 5 \%$ of ordinary matter. We have no idea of what is Dark Energy, we just know that we need it to explain the CMB data and that it could appear as a constant of the Einstein field equations in standard General Relativity (GR), with the effect of accelerating the expansion of the present Universe. A little more we know about Dark Matter, however we still lack a theoretical interpretation of the phenomenon, for example we still do not know if dark matter is composed of particle or if it is a modification of the Einstein's General Relativity equations. Moreover, about $7 \%$ of the ordinary matter is composed of neutrino, for which we still have many unanswered questions, like their mass, the hierarchy in which they exists or the possible existence of a invisible neutrino species with no interaction other than gravity (sterile neutrino).

In more than 50 years since the discovery of the $\mathrm{CMB}$, cosmologists were able to understand many things about the Universe and were able to build a "standard model" for cosmology that it is able to describe more than 14 billion years of the Universe evolution with percentage precision up to the present day. Yet, there are many ingredients that still are missing a physical interpretation and are the previously cited Dark Energy and Dark Matter.

The scope of the Euclid satellite is to improve our understanding, or to find a definitive answer, to the problems exposed so far [2]. While the main objectives of the mission is to study the equation of state of the Dark Energy and the nature of Dark Matter, the data obtained by this satellite will open the possibility to study with unprecedented details some other physical phenomena like neutrino and modification of gravity.

In this proceeding we will give an overview of the problems that Euclid is targeted to study, the characteristic of the satellite, the consortium of scientists devoted to study its data and the expected level of precision on parameters estimation.

\section{The Dark Universe}

To describe the physical phenomena that are the object of the Euclid satellite mission we will use the results obtained by the Planck collaboration in their last legacy publication [1]. The reason of that is threefold: firstly, Planck data gives the more precise simultaneous measurements of all the six parameters of the standard (so called vanilla) $\Lambda$ - Cold Dark Matter model, secondly, Planck data represent the best knowledge we have of the Universe now and this will be true even at the start and during all of the Euclid mission, and finally during this conference Planck collaboration received a prize ${ }^{1}$, so using their results is the best way to celebrate this prize and their work.

\subsection{Dark Energy}

All we know about Dark Energy from CMB analysis is that it is needed an extra energy component, in a form of a constant in the Einstein's field equation, to reconcile the observed power spectrum to the theoretical $\Lambda \mathrm{CDM}$ calculated power spectrum. To go beyond the cosmological

${ }^{1} 2019$ Giuseppe and Vanna Cocconi Prize for an outstanding contribution to Particle Astrophysics and Cosmology is awarded to the WMAP and Planck Collaborations 
constant interpretation of the dark energy, a phenomenological parametrization is build that allow a "time variation" of the Dark Energy equation of state $w$. So the standard Dark Energy equation of state $w=-1$ become a constant value plus a function of time (the scale factor $a$, or redshift) that is $w(a)=w_{0}+(1-a) w_{a}$. With this parametrization, the usual cosmological constant description is obtained setting $w_{0}=-1$ and $w_{a}=0$.

The most stringent constraints obtained by the Planck collaboration comes from a combination of Planck CMB and Lensing data and Large Scale Structure (LSS) data (that includes, Barionic Acoustic Oscillations, Redshift Space Distortion, Weak Lensing and Supernovae). Depending on how and which data are combined the Planck collaboration finds that both a cosmological constant or a small time variation are allowed by the data, yet the second, with low statistical significance.

\subsection{Dark Matter}

Planck yield the most precise measurement to date of the matter density of the Universe, and as in the case of Dark Energy, the cosmological model needs a matter density much higher that the density estimated from "weighting" the luminous matter and gas: again, to reconcile theory with the measured power spectrum of the CMB fluctuations we need an extra dark component, in this case Dark Matter.

To understand the nature of Dark Matter is possible to assume some models and try to see if the data of Planck reconciles with the data of the various direct or indirect Dark Matter search experiments. Depending on the model assumed Planck give some possible hints on the particle nature of the Dark Matter concordant with other experiments, yet again with low statistical significance.

\subsection{Neutrino}

To complete the missing information on neutrino model is one of the last open question of what lies beyond the Standard Model of Particle Physics. With Planck CMB data it was realized that it is possible to answer some of those open questions, in particular to measure the sum of neutrino masses, to select the neutrino mass hierarchy, and to answer about the possible existence of some sterile neutrino.

Assuming a value of $N_{\text {eff }}=3.046$ equal to the standard picture of neutrino (no sterile neutrino allowed) Planck was able to give a limit on the sum of neutrino masses, that is $\sum m_{v}>200 \mathrm{meV}$. This is the most precise constraints of neutrino masses up to date by an experiment alone, even compared to some direct neutrino mass measurement experiments.

Assuming an upper limit of the sum of neutrino masses, Planck was able to estimate the parameter $N_{\text {eff }} \sim 3.046$ with percent precision, excluding the presence of sterile neutrino affecting Cosmology.

\subsection{Modified Gravity}

A model to rule them all. All of the three above physical phenomena, Dark Energy, Dark Matter and Neutrino, can be explained (or have degeneracy, like in the case for Neutrino) with models of Modified Gravity (MG). As for the case of Dark Energy, it is possible to construct a model with three additional phenomenological parameters. Measured deviation of those parameters form the value obtained from standard General Relativity indicates that modification of general 
relativity is possible. This phenomenological model and the constraints on those parameters are showed in [1]. Again, the Planck's cMB data shows a window of possible deviation from GR, but with low statistical significance.

\section{The Euclid mission}

The European Space Agency (ESA) Euclid mission was selected with the primary objective to understand the properties and nature of Dark Energy, but it is capable to shed light on all the Dark Universe open questions described so far.

\subsection{The Euclid Consortium}

The data collected by the Euclid satellite will be carried out by a European-led consortium, the Euclid Consortium (EC), of more than 2000 people in over 100 laboratories in 15 countries. The Euclid Consortium is also responsible for the construction of the instrument payload of the Euclid satellite and for the development and implementation of the Euclid Consortium Science Ground Segment (EC-SGS) which will process all data collected by the satellite, and for the scientific exploitation of the processed data.

\subsection{The Euclid satellite main characteristics}

In order to study the Dark Universe, the satellite mount two instruments: the NISP (Near Infrared SpectroPhotometer) and the VIS (Visible InStrument), the first, is capable of measuring the photometric redshift of few billion and the spectra of tens of millions of galaxies, while the second, is able to photograph the shape of billions of galaxies, with both the instruments working at the same time in the same, large ( $\sim 30 \%$ free sky fraction), field of view. The light that enlighten the NISP and VIS instruments is collected by a 1.2m, 3-mirror anastigmatic Korsch telescope.

\subsection{VIS - Visible InStrument}

The VIS instrument consist of a camera operating at visible wavelengths (550-920 nm) made of a mosaic of $6 \times 6 \mathrm{e} 2 \mathrm{v}$ Charge Coupled Detectors, containing 600 million pixels, allowing measurements of the deformation of galaxies (shapes).

For about 1 billion galaxies Euclid will provide information at the same time on both on the shapes and the photometric redshifts, in a range of redshift $z \in[1,2]$. Moreover, for about 50 million objects galaxies Euclid will provide information on the same time on both shapes and spectroscopic redshifts (that is vastly more precise than the photometric redshift), providing the most accurate catalog of galaxies, capable of measuring at the same time, BAO, Redshift Space Distortion and Weak Lensing, and so provide a huge amount of cosmological information needed to study the elusive signals of Dark Energy, Dark Matter and neutrino.

\subsection{NISP - Near Infrared SpectroPhotometer}

The NISP instrument is a camera composed of a mosaic of $4 \times 4$ Teledyne H2RG detectors sensitive to near-infrared light radiation (1000-2000 nm) with 65 million pixels.

This setup will provide low precision measurements of redshifts, and thus distances, of over a billion galaxies from multi-color photometry; using a spectrometer to analyse the spectrum of light 
in near-infrared (1000-2000 nm), to acquire precise redshifts and distances of million galaxies, with an accuracy 10 times better than photometric redshifts, and to determine the Baryon Acoustic Oscillations (BAO).

The light of each galaxy will be measure by 3 broad band filters $\mathrm{Y}, \mathrm{J}$ and $\mathrm{H}$ that combined with 4 additional filters from ground experiments will provide the extraordinary accuracy on photometric redshift needed to study the time evolution of Dark Energy.

\section{Expected Results}

In December 2018, Euclid passed its critical design review, which validated the overall spacecraft design and mission architecture plan, and final spacecraft assembly was allowed to commence. This was a fundamental step to both confirm the nominal mission characteristic stated in [2] and to update the expected results with new findings from the one calculated in 2011.

\subsection{Euclid Timeline}

Euclid will be launched on a Soyuz rocket from Kourou at the end of 2022. Following a travel time of 30 days it will travel to the Sun-Earth Lagrangian point L2. During its nominal mission, which will last at least 6 years, Euclid will observe about 15,000 $\mathrm{deg}^{2}$, or about $\sim 1 / 3$, of the extra-galactic sky (the sky facing away from the Milky Way), and will have three data release with increasing number of $\mathrm{deg}^{2}$ covered.

\subsection{Forecasted Precision on some parameters}

In this final section we will review the forecasted precision of the parameters of interest to the Euclid mission objectives. Based on the original forecast of [2] we can now update their values with the new forecast of the Euclid Consortium [3]. The parameters and their precision are showed in Table 1.

\begin{tabular}{||ccc||}
\hline & Dark Energy & Modified Gravity \\
\hline Parameters & $w_{0}, w_{a}$ & $\gamma$ \\
\hline \hline $\mathrm{GC}_{s}$ & $0.25,0.98$ & 0.26 \\
$\mathrm{WL}$ & $0.22,0.96$ & 0.82 \\
$\mathrm{GC}_{s}+\mathrm{WL}$ & $0.084,0.33$ & 0.067 \\
\hline
\end{tabular}

Table 1: Euclid satellite optimistic expected precision (for non-flat cosmology) on some parameters of interest for different Euclid probes. $\mathrm{GC}_{s}$ stand for spectroscopic Galaxy Clustering and WL for Weak Lensing.

The results showed in Table 1 tell us that the $\mathrm{GC}_{s}$ and WL data are both capable of measuring deviation of the $w_{0}$ parameters from -1 at more than 3 -sigma precision. It is however only with the combination of the two that it is possible to constraint the time variation parameter $w_{a}$ on the required 3-sigma level precision.

For Modified Gravity we considered the precision with respect to the phenomenological growth index parameter $\gamma$. For standard General Relativity the value of $\gamma \sim 0.55$, so confronting with Table 1 we can see that only with the combination of the two probes $\left(\mathrm{GC}_{s}+\mathrm{WL}\right)$ it is possible to measure deviation of GR for more than the required 3-sigma. 
To consider the impact of neutrino precision for Euclid we will use use a new reference [4], where it is stated that "under very agnostic assumptions about non-linearities and short-scale physics a future Euclid-like survey will be able to measure the sum of neutrino masses with a standard deviation of $28 \mathrm{meV}$ ", that confronted to the actual most precise measurement coming from planck of about $200 \mathrm{meV}$ there is an improvement factor of about 10 for the sum of neutrino masses estimation, coming from Euclid alone. The quoted forecast indicates that the Euclid constraint on the sum of neutrino masses will be the most precise bound with respect to any direct measurements of neutrino mass coming from direct neutrino mass measurement for the next decade.

\section{Conclusion}

In those proceedings we showed some pressing modern open questions in Cosmology and how the Euclid mission is going to answer to those questions. We describe the characteristic of the Euclid satellite instruments and showed the precision expected on parameters estimation from those instruments. The forecasted values gives us hope to constrain with the greatest precision, possible deviation of the standard $\Lambda \mathrm{CDM}$ model with the Euclid data alone, and show importance for the fundamental physics studies of a cosmological experiment like Euclid.

\section{References}

[1] Planck Collaboration, N. Aghanim, Y. Akrami, M. Ashdown, J. Aumont, C. Baccigalupi et al., Planck 2018 results. VI. Cosmological parameters, arXiv e-prints (2018) arXiv:1807.06209 [1807 . 06209 ].

[2] R. Laureijs, J. Amiaux, S. Arduini, J. L. Auguères, J. Brinchmann, R. Cole et al., Euclid Definition Study Report, arXiv e-prints (2011) arXiv:1110.3193 [1110.3193].

[3] Euclid Collaboration, A. Blanchard, S. Camera, C. Carbone, V. F. Cardone, S. Casas et al., Euclid preparation: VII. Forecast validation for Euclid cosmological probes, arXiv e-prints (2019) arXiv:1910.09273 [1910.09273].

[4] A. Chudaykin and M. M. Ivanov, Measuring neutrino masses with large-scale structure: Euclid forecast with controlled theoretical error, arXiv e-prints (2019) arXiv:1907.06666 [1907. 06666 ]. 